Industries laitières, groupant et coordonnant, sur le plan national, les différentes disciplines indispensables, les recherches de laboratoire et leurs applications, dans les domaines variés mais complémentaires de la physiologie, de la physico-chimie, de la biochimie, de la technique et de l'Economie.

Un tel organisme devrait dominer et diriger des centres d'étude régionaux et posséder des laboratoires mobiles.

L'Industrie laitière française et sa législation ne devraient pas craindre de modifier certaines conceptions désuètes, d'adopter sans tarder des voies nouvelles, sous peine de demeurer en position seconde dans l'industrie laitière mondiale, après en avoir été, grâce à des avantages naturels, le chef de file au début $d u \mathrm{xx}^{\mathrm{e}}$ siècle.

Si une production et une utilisation rationnelles de nos ressources sont la clef de voûte de l'édifice à reconstruire, l'exportation de nos fabrications est inséparable de la qualité, du rendement, et de l'adaptation aux conditions modernes. L'abaissement des prix de revient est conditionné par une utilisation totale des sousproduits et la création correspondante de nouvelles industries dérivées, déjà très prospères à l'Etranger.

Il est vrai de dire que l'industrie laitière devrait constituer un des piliers de l'Economie nationale française, mais il faut alors rejeter les solutions partielles et, cessant d'adopter des palliatifs, évoluer hardiment. Notre renouveau et notre salut sont à ce prix.

Paris, 31 décembre 1945.

\title{
RECHERCHE SUR LE COMPORTEMENT BIOCHIMIQUE ET PHYSIOLOGIQUE DE LA VITAMINE A DANS LA SÉCRÉTION LACTÉE
}

\author{
par \\ ANDRÉ FRANÇOIS \\ Ingénieur Chimiste I. C. P. \\ Ingénieur-Docteur \\ (Suite)
}

\section{CHAPITRE III}

\section{CONSERVATION DE LA VITAMINE A DANS LE LAIT}

La conservation de la vitamine $\mathrm{A}$ dans le lait est un problème d'importance pratique. Le lait livré à la consommation conserve-t-il son pouvoir vitaminique ? Le lactose du lait subit, en effet, rapidement la fermentation lactique. Or, on sait que la vitamine A est relativement moins stable en milieu acide qu'en milieu alcalin. 
Dans les expériences que nous avons effectuées au cours de ce travail, les échantillons étaient transportés de la ferme, située dans la région parisienne, au laboratoire. Il était important de connaître quelle destruction subissait la vitamine $\mathrm{A}$ au cours de la conservation du lait.

Nous avons étudié la teneur en carotène et en vitamine $\mathrm{A}$ des différents échantillons de lait conservé dans diverses conditions :

1. Conservation à l'obscurité, glacière $2^{\circ}$, sous azote.

2. Conservation à l'obscurité, glacière $2^{\circ}$, sous air.

3. Conservation à l'obscurité, à $20^{\circ}$, sous air.

4. Conservation à la lumière, à $20^{\circ}$, sous azote.

5. Conservation à la lumière, à $20^{\circ}$, sous air.

Les échantillons étaient conservés dans des fioles-poires de $500 \mathrm{~cm}^{3}$ en verre ordinaire.

Les courbes de la fig. VI représentent la variation des quantités de carotène et de vitamine $A$, en $\%$ de la teneur initale.

On constate que le meilleur mode de eonservation de la vitamine A est celui qui consiste à maintenir le lait à l'obscurité, à basse température, sous azote. Après 50 jours de conservation dans ces conditions, il reste environ $78 \%$ du carotène et $76 \%$ de la vitamine $\mathrm{A}$.

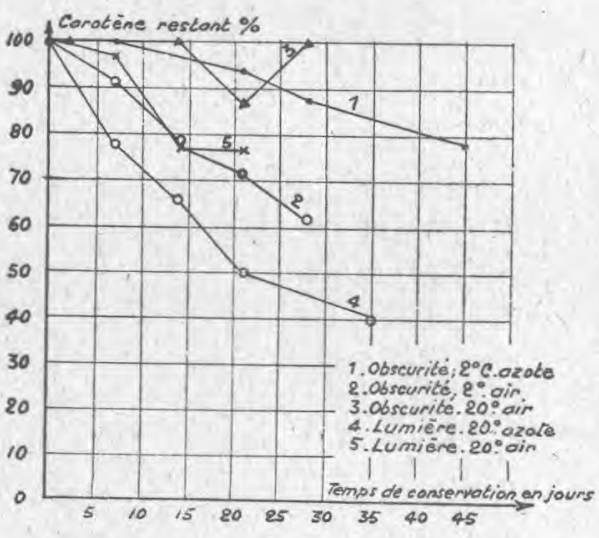

EIG. VI $a$

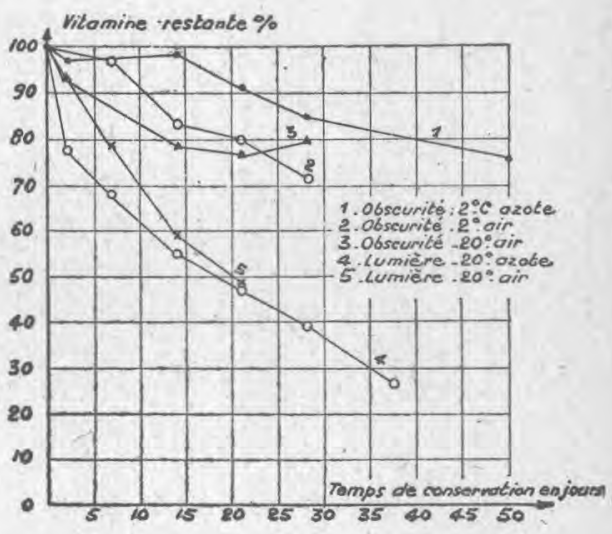

FIG. VI $b$

La destruction de la vitamine $A$ ne commence qu'après 15 jours de conservation. A l'obscurité, le carotène se conserve bien à $20^{\circ}$ sous air.

Nous avons voulu étudier la part qui revient à la température, à l'éclairement et à l'aération dans la conservation de la vitamine $\mathrm{A}$.

1. La conservation sous air à $2^{\circ}$, à l'obscurité (courbe 2 ) pro- 
voque une perte plus rapide en vitamine $\mathrm{A}$ et en carotène que la conservation sous azote (courbe 1 ).

2. La comparaison des courbes 2 et 3 indique qu'une élévation de température de 2 à $20^{\circ}$ n'accélère presque pas la destruction de la vitamine $\mathrm{A}$ du lait; le carotène semble ne pas être détruit à $20^{\circ}$ (les autres conditions étant : obscurité, air).

Il y a cependant acidification et caillage des échantillons conservés à $20^{\circ}$.

3. La comparaison des courbes 3 d'une part, 4 et 5 d'autre part, montre le rôle primordial joué par la lumière dans la destruetion de la vitamine $\mathrm{A}$ du lait.

Dans l'échantillon 3, conservé à l'obscurité, le carotène est tombé, en 21 jours, à $87 \%$ de sa teneur initiale, la vitamine A à $77 \%$ de sa teneur initiale, alors que dans l'échantillon 4 , conservé à la lumière, le carotène est tombé à $50 \%$, la vitamine $\mathrm{A}$ à $47 \%$ seulement de sa teneur initiale.

Dans l'échantillon 5, conservé dans les mêmes conditions de température $\left(20^{\circ}\right)$ que 3 et 4 , mais sous azote, la teneur en carotène atteint $77 \%$ de la teneur initiale et $47 \%$ de la teneur initiale pour la vitamine $\mathrm{A}$.

Ainsi, la vitamine $\mathrm{A}$ et le carotène contenus dans un milieu opaque, comme le lait, sont encore sensibles à l'action de la lumière.

La comparaison des courbes de la fig. 6 montre l'influence de la conservation sous azote $\left(20^{\circ}\right.$ à la lumière).

Les résultats sont sensiblement identiques dans les 2 cas quant à la vitamine A. La destruction est toutefois plus rapide lorsque le lait est conservé sous azote. Le carotène semble également détruit plus vite lorsque le lait est conservé sous azote.

Comment expliquer cette destruction ? Il est possible que la fermentation lactique, plus active en anaérobiose, cause la destruction de la vitamine A. A $2^{\circ}$, la fermentation lactique est entravée et c'est le contact de l'air qui oxyderait la vitamine A. On conçoit alors que l'atmosphère d'azote à cette température de $2^{\circ}$ retarde la destruction de la vitamine $\mathrm{A}$. Les produits de destruction de la vitamine A ne sont peut-être pas les mêmes si l'on conserve le lait sous azote et sous air.

Nous avons tenté de supprimer l'acidification et le «caillage» du lait par addition de diverses substances.

a) Bicarbonate de sodium.

Le bicarbonate de sodium n'empêche pas la fermentation lactique mais neutralise l'acide lactique au fur et à mesure de sa production. 
Les courbes 6 et 5 de la fig. VII montrent la destruction comparée de la vitamine A dans le lait conservé respectivement avec et sans bicarbonate. On voit que l'addition de bicarbonate de sodium, loin d'empêcher la destruction de la vitamine $A$, l'accélère au contraire.

Il se développe une fermentation putride plus néfaste à la vitamine $\mathrm{A}$ et au carotène

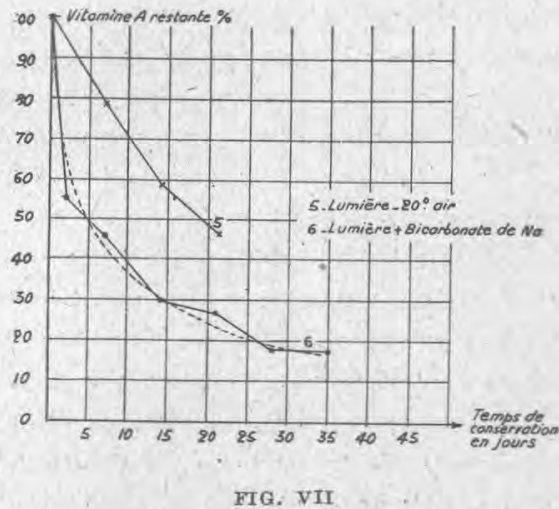
que l'acidification.

La courbe qui traduit la destruction (fig. VII) présente une allure remarquablement régulière, comme celle d'une réaction régie par la loi d'action de masses. La teneur finale en vitamine A est de l'ordre de celle du lait écrémé et semble s'y stabiliser.

\section{b) Addition de trioxyméthylène.}

Le trioxyméthylène est un antiseptique qui empêche toute fermentation. Le lait se conserve indéfiniment sans s'acidifier. En ce qui concerne la conservation de la vitamine A, le trioxyméthylène donne des résultats assez surprenants.

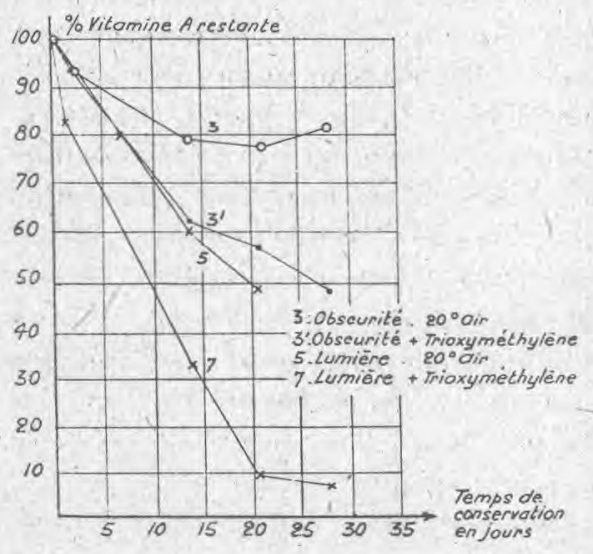

FIG. VIII

La comparaison des courbes 3 et 3 , 5 et 7 de la fig. VIII montre que le trioxyméthylène ne favorise pas la conservation de la vitamine A dans le lait ; au contraire, il accélère la destruction. La destruction est particulièrement rapide dans l'échantillon conservé à la lumière.

$\mathrm{Au}$ bout de 28 jours, il reste dix fois moins de vitamine A dans l'échantillon conservé à la lumière que dans celui conservé à l'obscurité.

Ainsi, au danger que présente la consommation de lait conservé à l'aide de bicarbonate de sodium ou de trioxyméthylène, s'ajoute l'inconvénient grave de la destruction rapide de la vitamine A. 
On ne saurait trop souligner le caractère nocif des laits conservés au trioxyméthylène ou au formol. On sait que la formaldéhyde dénature les protides en bloquant leurs fonctions amines. Le tube digestif risque d'être ainsi gravement lésé.

En résumé, les meilleures conditions de conservation du carotène et de la vitamine $\mathrm{A}$ dans le lait sont : $1^{\circ}$ maintenir l'échantillon à l'obseurité (condition la plus importante) à basse température, sous azote, cette dernière condition étant à respecter uniquement lorsque le lait est conservé à basse température ; $2^{0}$ la conservation sous azote ne s'impose pas à la température ordinaire.

Dans les conditions industrielles pratiques, le lait mis en chambre froide et transporté en bidons opaques, dès sa production, ne perd pratiquement pas son activité vitaminique $\mathbf{A}$.

\section{CHAPITRE IV \\ ETUDE PHYSIOLOGIQUE}

\section{INTRODUCTION}

Pour étudier la physiologie de la vitamine A dans la glande mammaire, il eut été très instructif d'étudier d'une part le sang artériel et le sang veineux irrigant la glande mammaire, et d'autre part le lait, quant à leurs teneurs en carotène et en vitamine A.

Un bilan du carotène et de la vitamine $\mathrm{A}$ dans chacun de ces trois milieux nous aurait permis d'étudier le sort de ces corps dans la glande mammaire.

On sait malheureusement peu de choses sur le rôle que peut jouer à cet égard le système lymphatique. Il doit être cependant d'une grande importance, puisque le lait emprunte au sang ses éléments constitutifs par' l'intermédiaire de la lymphe tissulaire. L'étude du sang veineux et artériel n'a cependant pas été possible.

En dehors des difficultés pratiques, cette étude est impossible en raison même de l'approximation insuffisante donnée par le dosage de la vitamine A. En effet, il est admis qu'un litre de lait est formé à partir de 400 litres de sang. L'appauvrissement du sang à son passage dans la mamelle sera de $1 / 400^{\circ}$ pour chacune des substances qui sont des précurseurs des constituants du lait. La précision du dosage de la vitamine A est bien inférieure à cette limite, et cette étude n'aurait eu aucune signification.

Il faudrait que l'appauvrissement soit de l'ordre de $10 \%$ pour que des conclusions puissent être tirées.

Mais il existe un produit de l'activité de la glande mammaire qui présente des caractères qui le rapprochent à la fois du sang et du lait : le colostrum. C'est une sécrétion lactée subnormale qui met en relief, en les exagérant, certains phénomènes du passage de la vitamine $A$ du sang au lait. 
TABLEAU VIII

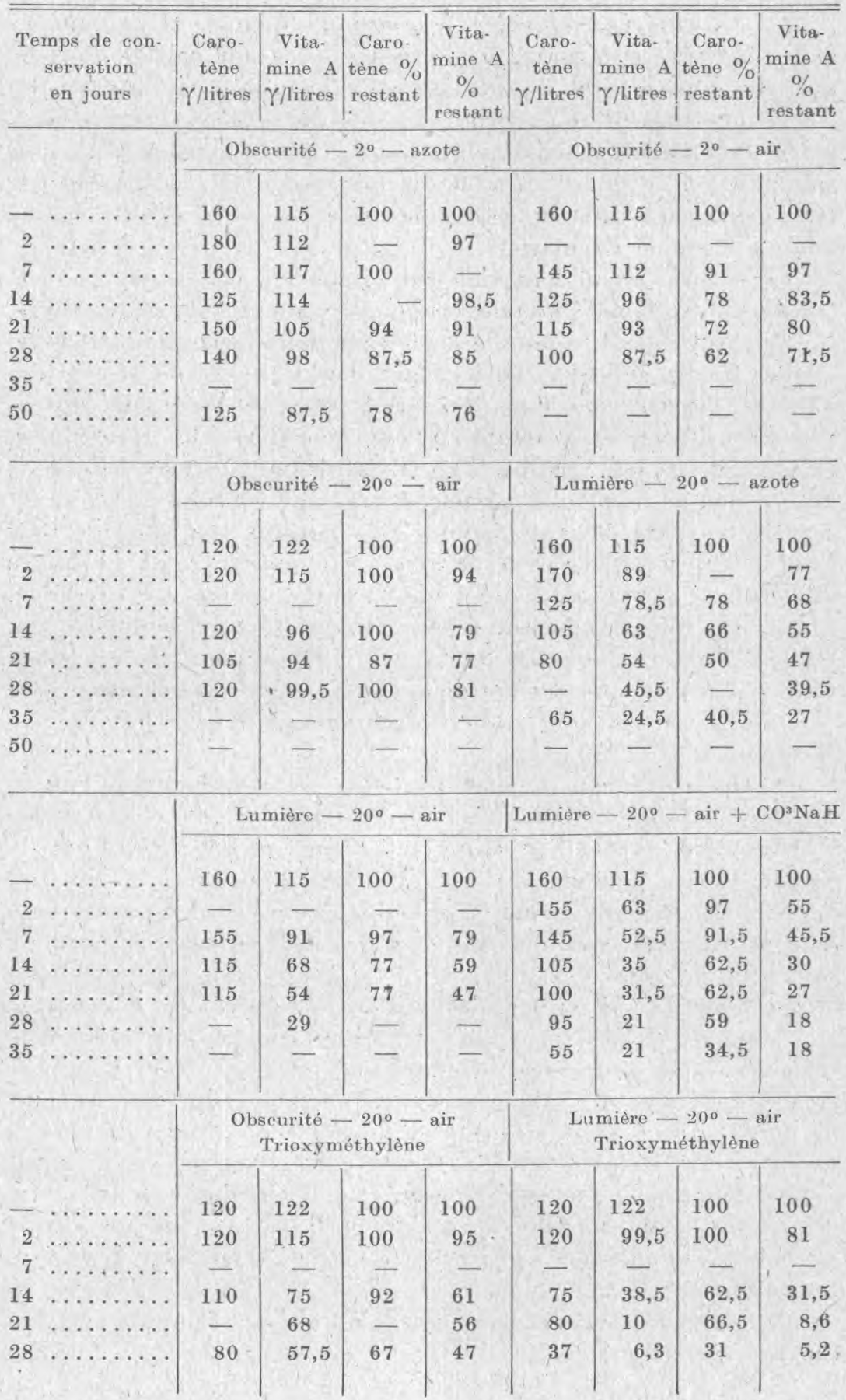




\section{Le sang}

A. - L'alimentation végétale fournit à l'homme et aux animaux des quantités importantes de carotène, qui subit une destruction partielle dans le tube digestif. Il passe ensuite dans l'intestin où il subit une absorption partielle. Avee les acides biliaires, et en partieulier avec l'acide désoxycholique, le carotène forme un complexe soluble dans l'eau et capable de traverser la paroi intestinale (Greaves et Schmidt, Euler et Klussmani). Il passe alors dans le système circulatoire.

Là encore, on ne sait rien sur le rôle de la lymphe dans le transit de carotène. Une telle étude est évidemment très malaisée.

Le carotène existe donc dans le sang sous forme hydrosoluble.

Les lipides subissent un sort parallèle sous l'action des acides biliaires. On sait que, dans le sang, la majeure partie des lipides existe sous forme hydrosoluble, à l'état de cénapses lipo-protéiques. (MACHebGUF [55], SANDOR [51]). Le sérum est un milieu limpide, dans lequel on ne peut distinguer de globules graisseux.

D'autre part, il a été montré par Palmer [49] et Van Den BERGH [50] que le carotène du sérum sanguin est lié aux protides du sérum. Palmer a pu isoler une caroto-albumine hydrosoluble. Le cholestérol et la lécithine sont présents dans le complexe et, selon Palmer, en assurent la stabilité. Remarquons que le cholestérol et lécithine existent dans les complexes lipo-protéiques et que SANDOR pense que leur stabilité, plus grande en été, est due au carotène.

On est ainsi amené à constater l'extrême complexité de l'étude du carotène dans le milieu sanguin : ce pigment existe sous forme hydrosoluble, étant lié vraisemblablement au sel de sodium de l'acide désoxycholique, aux lipides et aux protides,

Il est intéressant de noter que ni le sérum ni la graisse de porc ne contiennent de carotène. Il y en a peu dans le lait et le sérum de chèvre et de brebis.

Le carotène mis en circulation dans le sérum sera transformé en vitamine A. Les recherches de Mooke ont montré que eette transformation s'effectue vraisemblablement dans le foie. Le récent travail de VINET et Collaborateurs [56] semble confirmer ce point de vue. Ce qui est sûr, e'est que la vitamine A est mise en réserve dans le foie. D'après Chevílirer [57], la vitamine A existerait dans le foie sous la forme ester, alors qu'elle circulerait dans le sang sous la forme alcool. Selon GuILBERT et Hart [58], les réserves de vitamine A de la vache en croissance seraient comprises entre 1 et 5 grammes.

Les cellules de KUPFER joueraient un rôle actif dans la fixation du carotène sanguin. 
La teneur du sang en vitamine A serait fonction de la réserve hépatique (Van ECKelen et Wolff [100]). MacCord et Clausen [101] semblent indiquer une dissociation nette entre ces deux valeurs. L'état infectieux peut également faire cesser cette relation (LindQvist [59]). Le travail de VARANGOT [60], sur l'homme, indique qu'une déficience dans la teneur du sang en vitamine A est bien un témoin de carence, les chiffres indiquant une relation entre cette quantité et la réserve hépatique. Ces résultats ont toutefois une valeur statistique. Il peut y avoir, d'un jour à l'autre, de grandes variations dans le taux sanguin de vitamine $\mathrm{A}$.

\section{B. - Relation entre la teneur du sang et celle du lait en vitamine A :}

Moore [61] a observé que la teneur du sang en carotène augmente très rapidement après la mise à l'herbe. Quelques heures après le changement d'alimentation, l'augmentation est déjà significative. Cet auteur trouve des teneurs différentes selon la race des animaux. Les teneurs croissent quand on passe des Brunes des Alpes aux Holstein, puis aux Ayrshires, puis aux Jersey et aux Guernesey.

Peterson et Collaborateurs [62] ont pu constater qu'il y avait un certain parallélisme entre la teneur du sérum en carotène et la teneur du beurre en carotène et en vitamine A. Cependant, SEmb, BAUMANN et STEENBOCK [63] trouvent que les hautes teneurs en carotène du colostrum sont indépendantes d'une augmentation du taux de caroténoïdes du sérum. NyLund, puis CHEvalLiter et Collaborateurs [64], chez la femme, ont remarqué que la teneur du sang en vitamine A est généralement supérieure à celle du lait; chez la chienne, ces derniers auteurs ont trouvé que le lait avait une concentration en vitamine $\mathrm{A}$ supérieure à celle du sang. La concentration du carotène dans le 'sérum sanguin est d'ailleurs sujet à de grandes variations individuelles. LELESz [78] a trouvé dans le sérum de vache des valeurs s'étageant de 51 à $268 \mathrm{U}$. I. pour $100 \mathrm{~cm}^{3}$ de sérum. Les résultats de DAvis et MADSEN [65] montrent que la teneur du sang en vitamine A dépend étroitement de la quantité de carotène apportée par la ration actuelle, et aussi des réserves emmagasinées par l'animal. La teneur en vitamine A du plasma est étroitement liée à la teneur en carotène. Cependant, la teneur en vitamine A n'augmente pas proportionnellement aux quantités de carotène distribuées aux animaux.

\section{Travail personnel}

Au cours d'une période de deux semaines (15-30 décembre), nous avons étudié parallèlement les teneurs du sang et du lait de vaches en vitamine $A$. 
Les animaux étaient divisés en trois lots recevant chacun une quantité différente de foin $(*)$. Chacun de ces lots était lui-même divisé en deux groupes, dont l'un recevait de la vitamine A, l'autre n'en recevait pas.

Le tableau ci-dessous résume les résultats :

TABLEAU IX

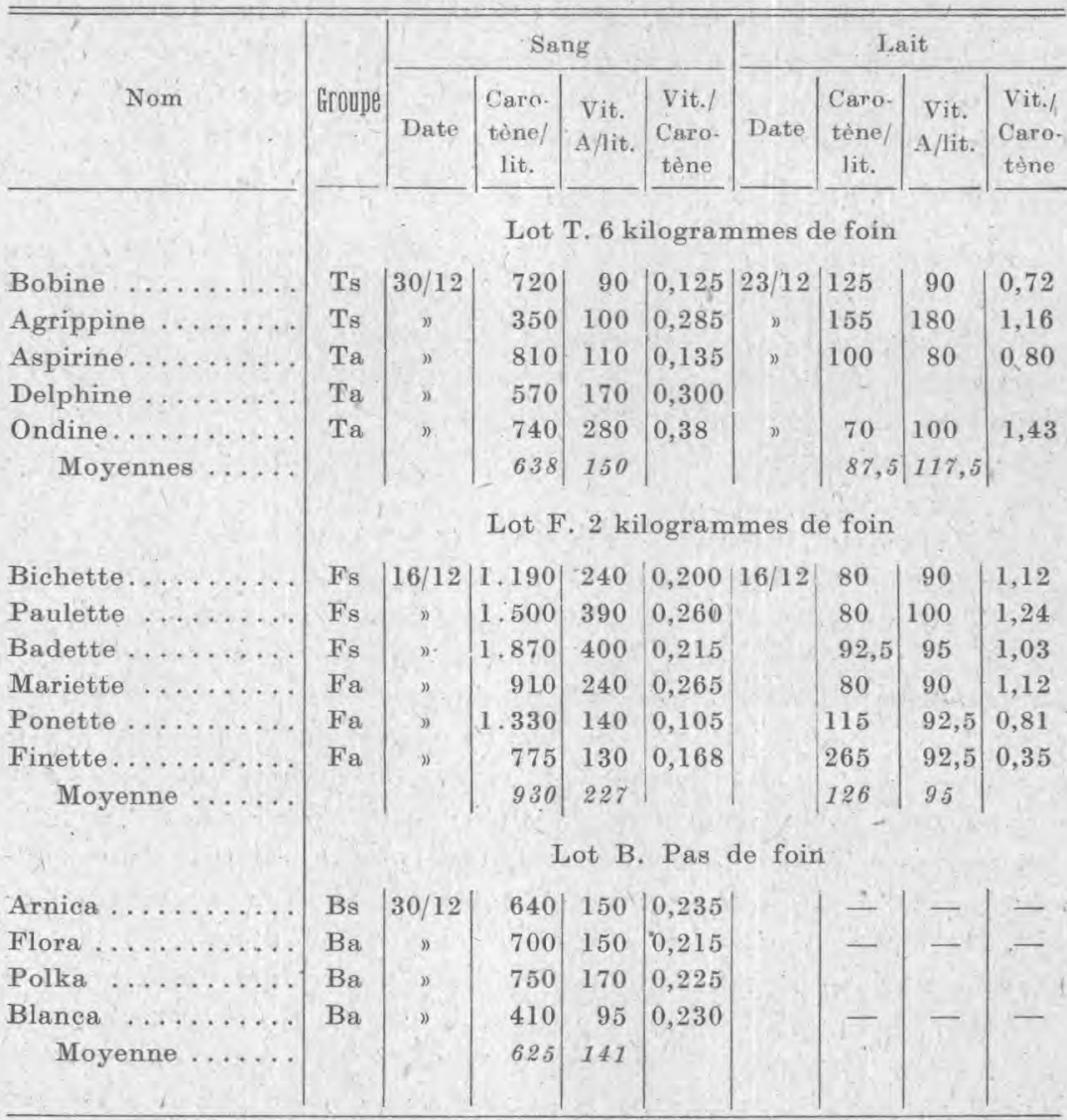

L'indice a indique que les animaux recevaient de la vitamine A.

L'indice s indique que les animaux n'en recevaient pas.

On voit que l'ingestion d'une quantité de $6 \mathrm{~kg}$. de foin n'a pas augmenté la teneur du sérum sanguin en carotène et en vitamine $A$. Cette teneur est la même - la différence étant de l'ordre de l'erreur expérimentale - que la teneur du sérum de vaches ne recevant pas de foin.

Nous verrons plus loin que la teneur du lait en carotène et en

(*) Le foin contenait $14 \mathrm{mg}$. de carotêne par kilogramme. 
vitamine n'est pas sensiblement augmentée par l'absorption de $6 \mathrm{~kg}$. de foin.

Le sérum des vaches du lot $\mathrm{F}$, ne recevant cependant que $2 \mathrm{~kg}$. de foin, présente des teneurs en carotène et en vitamine $\mathrm{A}$ plus élevées que celui des vaches des autres lots. Nous pensons qu'il faut en voir la raison dans le fait que les sangs et les laits des vaches de ce lot ont été prélevés deux semaines plus tôt que les autres : les animaux, pendant ces deux semaines, se sont déchargés de leurs réserves vitaminiques.

Les variations individuelles dans les teneurs du sang en facteurs A sont assez considérables. Les résultats montrent que les teneurs en carotène et en vitamine A sont parallèles dans le sérum. Les vaches du lot $\mathrm{F}$ présentent en effet des teneurs plus élevées à la fois en carotène et en vitamine $A$. Cependant, le lait produit par ces vaches n'est pas plus riche en vitamine A que celui produit par les vaches du lot $\mathrm{T}$ ( $6 \mathrm{~kg}$. de foin).

La teneur moyenne en carotène est légèrement augmentée, mais il y a cependant des variations individuelles dans le sens inverse.

Remarquons que les valeurs $d u$ rapport vitamine $A /$ carotène sont d'autant plus dispersées que les vaches reçoivent plus de carotène. Il est remarquablement constant chez les vaches du lot B ne recevant pas de foin. Ce fait est dû à l'assimilation différente du carotène par chaque individu.

Le rapport vitamine $A$ /carotène est plus grand dans le lait que dans le sang. L'épithélium mammaire laisserait passer plus facilement la vitamine A que le carotène, ou bien il faudrait envisager une transformation du carotène en vitamine A dans la mamelle.

Pour des animaux soumis à une même alimentation ou à une alimentation dont la teneur en carotène reste inférieure à un certain seuil, la teneur du sang en facteurs A n'a pas d'influence sur celle du lait. Cependant, lorsque les vaches sont au pâturage, la teneur du sang en facteurs $A$ est très augmentée, ainsi que celle du lait ou du colostrum.

Par exemple, nous avons effectué des prélèvements simultanés de sang et de colostrum sur des vaches soumises à une alimentation différente. Les prises étaient effectuées 3 ou 4 heures après vêlage.

La vache I recevait $7 \mathrm{~kg}$. de foin, la vache II était au pâturage.

\begin{tabular}{|c|c|c|c|c|c|}
\hline \multirow[t]{2}{*}{ 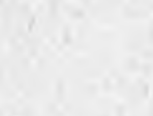 } & \multicolumn{2}{|c|}{ Colostrum } & \multicolumn{3}{|c|}{ Seng (Plasma) } \\
\hline & $\begin{array}{c}\text { Carotène } \\
\gamma / \text { litre }\end{array}$ & $\begin{array}{r}\text { Vitamine } \\
\gamma / \text { /litres }\end{array}$ & A & $\begin{array}{c}\text { Carotène } \\
\alpha / \text { litres }\end{array}$ & $\begin{array}{r}\text { Vitamine } \\
\alpha / \text { litres }\end{array}$ \\
\hline I & 730 & 1,370 & & 330 & 146 \\
\hline 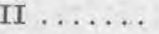 & 2.440 & 1.900 & & 2.380 & 610 \\
\hline
\end{tabular}

L'augmentation du taux de carotène est plus grande que celle qui concerne la vitamine $\mathrm{A}$. 
Il semble - et d'autres faits viendront appuyer cette hypothèse - que le carotène ne puisse être mis en réserve par l'organisme.

Le tableau IX montre que les teneurs respectives en vitamine A du sang sont en général plus grandes que celles du lait.

La concentration du colostrum en vitamine A est très différente de celle du lait. Les taux de carotène dans chacun de ces deux milieux sont plus voisins.

Il semble que pendant la période colostrale, l'organisme ait un pouvoir aceru de transformer ee carotène en vitamine A. En effet, la concentration de la vitamine A dans le colostrum est bien supérieure à celle du sang.

Si le colostrum était un lait de rétention, la disparition de la vitamine $\mathrm{A}$ ne serait-elle pas plus rapide que celle du carotène ?

\section{LE COLOSTRUM}

1. Il y a lieu de distinguer le colostrum ante-partum et le colostrum post-partum. Nous étudierons ici uniquement ce dernier.

C'est la sécrétion de la glande mammaire qui apparaît immédiatement après la parturition et dont la composition tend vers celle du lait. Sa composition chimique en est très différente et varie rapidement après le part.

L'amplitude des variations est très importante pour certains constituants, en particulier pour les protides, et plus spécialement pour la globuline, pour le carotène et pour la vitamine A.

Sans entrer dans les détails, voici les caractéristiques principales du colostrum, d'après EngEL et Schlag [66] : $1^{\circ}$ la composition des colostra, leurs teneurs en éléments, sont spécifiquement différentes chez les vaches prises individuellement; $2^{\circ}$ l'acidité diminue et devient normale après $6-12$ jours ; $3^{\circ}$ l'essai de cuisson n'est plus positif lorsque l'acidité est de $10^{\circ}$, sans pour cela que la composition chimique soit devenue normale ; $4^{\circ}$ le poids spécifique après la misebas est de 1.060 à $1.080 ; 5^{\circ}$ la réfraction du sérum est faible ; $6^{\circ}$ la teneur en lactose augmente; $7^{\circ}$ la teneur en chlorures diminue ; $8^{\circ}$ l'abaissement du point de congélation est très irrégulier; 90 la teneur en matières protéiques solubles est très élevée; $10^{\circ}$ la teneur en matière sèche est très élevée : 25,1 à $33,6 \% ; 11^{\circ}$ la teneur en matière grasse oscille, mais est plus élevée que dans le lait. Elle est plus faible quand on la rapporte à l'extrait sec; $12^{\circ}$ la teneur en cendres est plus élevée que dans le lait, mais moindre si on la rapporte à l'extrait sec ; $13^{\circ}$ la teneur en chaux et en magnésie varie beaucoup ; $14^{\circ}$ la période colostrale dure environ 12 jours (les protéines sont devenues normales).

Les teneurs en amino-acides, iode, cuivre, fer, arsenic, hormones 
et diastases sont également plus grandes que dans le lait (voir Houdinik̀Re [67]).

Il en est de même pour les vitamines BI, C, D, et surtout pour la vitamine $\mathrm{A}$ et le carotène. Plusieurs auteurs ont signalé que le colostrum est plus riche en vitamine A que le lait normal. DANN [37], Semb, Baumann et Steenbock [63], Guilbert et 'Hart [58], Leuschen, Kunerth, Kramer, Riddel [68]. Dann signale quele colostrum peut être de 10 à 100 fois plus riche en vitamine A que le lait normal. La chute est très rapide les premiers jours et redevient normale du cinquième au dixième jour. Stewart et MacCallum [69], étudiant les colostra de 100 vaches en stabulation, trouvent des valeurs s'étageant entre 35 et 1.181 unités internationales pour $100 \mathrm{~cm}^{3}$.

Nous avons étudié un certain nombre de colostra en ce qui concerne leur teneur en carotène et en vitamine $\mathrm{A}$, et les influences qui peuvent modifier ces teneurs : alimentation, âge de l'animal, etc.

\section{Travail expérimental}

A. Allure générale du phénomène.

Nous avons d'abord étudié la teneur en carotène et en vitamine A de colostra de vaches placées dans différentes conditions.

Dans une première expérience, nous avons étudié les variations des teneurs en lipides, protides, carotène et vitamine $A$.

L'animal était une vache holland aise, âgée de 3 ans, primipare, vêlée le 3 mars 1943.

L'alimentation quotidienne moyenne était la suivante : pulpe ensilée, $80-90 \mathrm{~kg}$., foin de trèfle, $7 \mathrm{~kg}$.; tourteau d'arachide, $0 \mathrm{~kg}$. 500 . Le foin de trèfle, de mauvaise qualité, contenait 5 mgr. de carotène par kilogramme et apportait done quotidiennement $35 \mathrm{mgr}$. de carotène. La traite était effectuée 2 fois par jour ( 5 h. et 14 h. 30 ). L'échantillon de colostrum soumis à l'analyse était un échantillon moyen provenant du mélange de quantités proportionnelles à celles qui étaient reeueillies au cours des traites du matin et du soir.

Les dosages suivants étaient effectués le plus rapidement possible : lipides, protides, carotène, vitamine A. Les lipides étaient dosés par la méthode de Gerber, les protides par la méthode de Kjeldahl (azote total $\times 6,25$ ), le carotène et la vitamine A selon la technique décrite antérieurement. Les tableaux X et XI et les courbes des fig. IX à XVII, traduisent les résultats expérimentaux.

\section{B. Interprétation et discussion.}

Les quantités totales de lait sécrété, les quantités totales de protides et de lipides augmentent rapidement à mesure que la sécrétion lactée s'installe. Ceci est bien connu. 
TABLEAU $\mathrm{X}$

TENEURS PAR LITRE DE COLOSTRUM

\begin{tabular}{|c|c|c|c|c|c|c|c|c|}
\hline Date & $\begin{array}{c}\text { Jours } \\
\text { après vêlage }\end{array}$ & $\begin{array}{l}\text { Lipi- } \\
\text { des } \\
\text { Gr. }\end{array}$ & $\begin{array}{c}\text { Proti- } \\
\text { des } \\
\text { Gr. }\end{array}$ & $\begin{array}{l}\text { Caro- } \\
\text { tène } \gamma\end{array}$ & $\begin{array}{l}\text { Vit. A } \\
\text { U./li- } \\
\text { tre }\end{array}$ & $\begin{array}{c}\text { Vita- } \\
\text { mine } \Delta \\
\gamma\end{array}$ & $\begin{array}{l}\text { Caro- } \\
\text { tène } \\
\text { par } \\
\text { gram- } \\
\text { me de } \\
\text { lipide }\end{array}$ & $\begin{array}{c}\text { Vita- } \\
\text { mine A } \\
\text { par } \\
\text { gram- } \\
\text { mede } \\
\text { lipide }\end{array}$ \\
\hline $3-3-43$ & $\begin{array}{r}\text { Jour du vêlage } \\
1 \text { seule traite }\end{array}$ & 41 & 159,5 & 730 & 4.800 & 1.370 & 17,8 & 33 \\
\hline $4-3-43$ & 1 & 38 & 65,4 & 420 & 2.600 & 910 & 11 & 24 \\
\hline $5-3-43$ & 2 & 40 & 47,5 & 190 & 1.050 & 365 & 4,25 & 9,1 \\
\hline $6-3-43$ & 3 & 45 & 47,1 & 90 & 640 & 225 & 2 & 5 \\
\hline $9-3-43$ & 6 & 37 & 43,3 & 45 & 340 & 120 & 1,18 & 3,25 \\
\hline $12-3-43$ & 9 & 35,5 & - & 50 & 325 & 114 & 1,4 & 3,2 \\
\hline $16-3-43$ & 13 & 33,5 & 38,5 & 45 & 240 & 84 & 1,34 & 2,5 \\
\hline
\end{tabular}

TABLEAU XI

QUANTITÉS TOTALES

\begin{tabular}{|c|c|c|c|c|c|c|}
\hline Date & $\begin{array}{c}\text { Jours } \\
\text { après vêlage }\end{array}$ & $\begin{array}{c}\text { Lipides } \\
\text { Gr. }\end{array}$ & $\begin{array}{c}\text { Protides } \\
\text { Gr. }\end{array}$ & $\begin{array}{c}\text { Carotène } \\
\gamma\end{array}$ & $\begin{array}{c}\text { Vita- } \\
\text { mine A } \\
\gamma\end{array}$ & $\begin{array}{l}\text { Poids } \\
\text { de lait } \\
\text { Kg. }\end{array}$ \\
\hline 3-3-43 . & $\begin{array}{r}\text { Jour du vêlage } \\
1 \text { seule traite }\end{array}$ & 72,7 & 271 & 1. 240 & 2.340 & 1,700 \\
\hline $4-3-43$ & 1 & 338,2 & 582 & 3.740 & 8.100 & 8,900 \\
\hline $5-3-45$. & 2 & 513,2 & 609,4 & 2.440 & 4.700 & 12,830 \\
\hline $6-3-43$ & 3 & 646,6 & 676,8 & 2.190 & 3.210 & 14,370 \\
\hline 9-3-43, & 6 & 537,2 & 628,7 & 655 & 1. 740 & 15,520 \\
\hline $12-4-43$. & 9 & 566,9 & - & 800 & 1.820 & 15,970 \\
\hline $16-3-43$ & 13 & 580,5 & 667,2 & 780 & 1.820 & 17,330 \\
\hline
\end{tabular}

Mais pour le carotène et la vitamine $A$, le phénomène présente l'allure inverse. Les premiers jours, les quantités totales excrétées sont considérables et décroissent ensuite rapidement pour devenir sensiblement constantes au delà du sixième jour.

Les courbes qui représentent les variations des teneurs par litre affectent une allure identique pour les protides, le carotène et la vitamine A. Elles sont d'une régularité remarquable et GrIMMFR [77] a pu traduire cette variation des teneurs en protides totaux, en albumine et en extrait sec dégraissé par une loi mathématique. Il semble que le carotène et la vitamine A suivent la même loi.

L'allure est identique pour les variations des quantités totales 
et des quantités par litre en ce qui concerne le carotène et la vitamine A. Elle est différente pour les protides. La courbe qui représente la variation des teneurs en lipides est également différente.

Il n'y a pas parallélisme, dans le colostrum, entre la teneur en lipides et la teneur en facteurs A. Ce fait est bien mis en évidence par l'étude de la variation des quantités de carotène et de vitamine A par gramme de lipides de chaque échantillon. Les teneurs décroissent rapidement et atteignent un minimum en 6 jours pour le carotène ; en 13 jours, il ne semble pas encore atteint pour la vitamine A.

Au sujet de la décroissance rapide des teneurs du colostrum en protides, on remarque que la majeure partie de ces protides sont constitués par des holoprotéides solubles - et en particulier par la globuline. Or, il a été démontré (Crowtrher et RAISTRICK) (WOODMANN) que les globulines du sang, du colostrum et du lait sont identiques.

Il semble que ce sont les composants non élaborés par la mamelle qui sont les plus abondants dans le colostrum : chlorures, matières minérales, vitamines, alors que les composants synthétisés par la mamélle sont moins abondants (lactose, caséine).

Les propriétés physiques et chimiques de la matière grasse colostrale approchent celles de la graisse de l'animal (ENGEL, Schlag et MoHr [70], de Vries [71].

C. Influence de l'alimentation ante-partum sur la teneur du colostrum en carotène et en vitamine $A$.

Nous verrons plus loin qu'on ne peut comparer en toute rigueur que les teneurs de colostra de vaches ayant subi le même nombre de parturitions.

Comparons les teneurs en carotène et en vitamine $\mathrm{A}$ de deux vaches : l'une, hollandaise, âgée de 7 ans, à son quatrième vêlage, reçoit depuis trois mois au moins : betterave, son mélassé, c'est-àdire une alimentation presque totalement dépourvue de carotène.

L'autre, normande, est au pâturage depuis 3 mois, et de ce fait ingère une quantité considérable de carotène (de l'ordre de 100 grammes pendant ces 3 mois).

Rappelons les teneurs de chacune des premières traites :

$\begin{array}{rrc} & \begin{array}{c}\text { Carotènę } \\ \gamma \text { p./litre }\end{array} & \begin{array}{c}\text { Vitamine A } \\ \gamma \text { p./litre }\end{array} \\ \text { I } \ldots \ldots \ldots \ldots \ldots \ldots & 630 & 1.370 \\ \text { II } \ldots \ldots \ldots \ldots \ldots & 2.440 & 1.900\end{array}$

L'énorme quantité du carotène ingérée 3 mois avant vêlage a eu un effet somme toute assez minime, puisque la teneur du 
premier colostrum en vitamine A a été multipliée par 2,5 et la teneur en carotène par 3,9 , quand. la quantité absorbée passait de quelques décigrammes à 100 grammes.

La variation saisonnière dans la teneur du colostrum en vitamine A est beaucoup moins ample que dans le cas du lait; les teneurs peuvent en effet varier de 1 à 20 pour le carotène, de 1 à 10 pour la vitamine $A$, respectivement dans le lait d'hiver et le lait d'été.

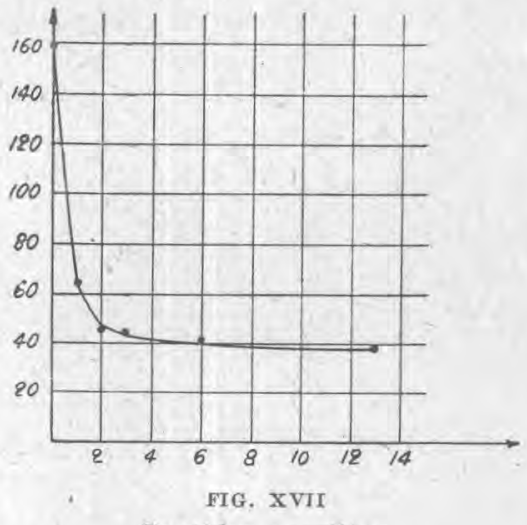

Protides par litre

L'organisme de la vache en gestation ne semble pas pouvoir mettre en réserve les quantités considérables de carotène ingéré ; ou bien, si ees réserves sont constituées, il existe un mécanisme physiologique qui ne permet pas l'excrétion du facteur $\mathrm{A}$ au delà d'une certaine limite.

Il semble d'ailleurs que la teneur du colostrum en carotène soit d'avantage influencée par l'alimentation que la teneur en vitamine.

Nous retrouverons le même phénomène à propos de l'étude du lait : les variations saisonnières des teneurs en carotène sont plus grandes que celles de la vitamine A : celle-ci semble être mise en réserve alors que le carotène serait rapidement excrété.

Considérons maintenant le cas d'animaux à l'étable depuis le 15 novembre, et recevant $6 \mathrm{~kg}$. de foin par jour. La vache Florine (deuxième parturition - 5 ans 1/2), vêlée le 9 décembre, recevait $6 \mathrm{~kg}$. de foin par jour une semaine avant vêlage (tableau XV).

La vache Perrine (deuxième parturition -4 ans), vêlée le 14 février, recevait $6 \mathrm{~kg}$. de foin 2 mois $1 / 2$ avant le part.

Les teneurs de la première traite sont les suivantes :

$\begin{array}{lrr} & \begin{array}{c}\text { Carotène } \\ \text { p./litre }\end{array} & \begin{array}{r}\text { Vitamine A } \\ \text { p. } / \text { litre }\end{array} \\ \text { Florine } \ldots \ldots \ldots \ldots & 2.080 & 1.630 \\ \text { Périne } \ldots \ldots \ldots \ldots & 860 & 1.900\end{array}$

Les teneurs en vitamine A sont bien du même ordre de grandeur mais c'est l'animal qui a reçu $6 \mathrm{~kg}$. de foin le plus longtemps qui donne le colostrum le plus pauvre en carotène.

Cependant, on remarque qu'avant le vêlage, Florine n'est restée en stabulation qu'à peine 1 mois, alors que Périne y restait 2 mois. Pendant ce temps, les réserves de earotène se sont rapidement 
éliminées - il est difficile de dire comment - puisqu'il n'y a pas eu excrétion par le lait. Peut-être, y a-t-il eu transformation en vitamine A et mise en réserve sous cette forme.

De même, les colostra des deuxièmes traites des vaches : Agrippine (4 ans - deuxième vêlage) et Biquette (4 ans - premier vêlage):

$\begin{array}{ccc} & \begin{array}{c}\text { Carotène } \\ \gamma p . / \text { litre }\end{array} & \begin{array}{c}\text { Vitamine A } \\ \gamma / \text { litre }\end{array} \\ \text { Agrippine } \ldots \ldots \ldots & 1.850 & 2.470 \\ \text { Biquette........ } & 1.065 & 2.245\end{array}$

Ici encore, les teneurs en vitamine A sont parfaitement comparables, et les teneurs en carotène différentes. Or, Agrippine recevait $6 \mathrm{~kg}$. de foin une semaine avant vêlage. Biquette ne recevait pas de foin.

Toutefois, la vache Biquette vêlait 1 mois plus tard.qu'Agrippine, ce qui pourrait également être la cause de la teneur en carotène plus faible.

B. Vitesse de variation de la teneur du colostrum en carotène et en vitamine $A$.

La fig. XVIII traduit les teneurs en fonctions du temps écoulé depuis le vêlage (en heures) des colostra dont les teneurs sont consignées dans les tableaux X, XII et XIII.

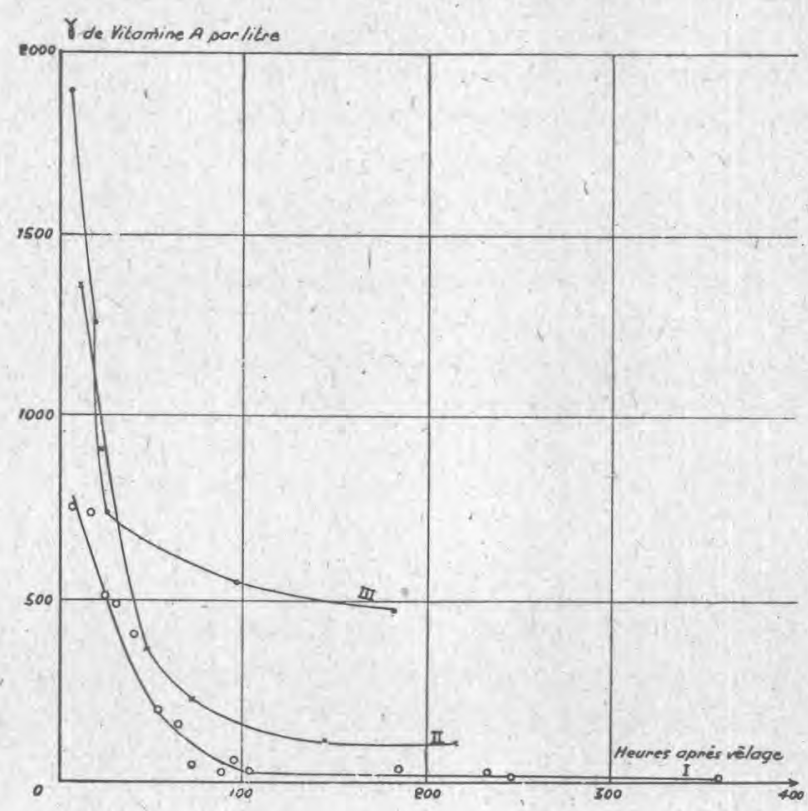

On peut ainsi comparer la variation pour une vache ne recevant pas de foin (courbe I), pour une vache recevant $7 \mathrm{~kg}$. de foin (courbe II), pour une vache au pâturage (courbe III).

La chute est extrêmement rapidedans tous les cas; les teneurs atteignent pratiquement celles du lait 100 heures après vêlage. IIG. XVIII Elles se fixent à 
TABLEAU XII

VACHE $\mathrm{N}^{\circ} 1$

\begin{tabular}{|c|c|c|c|c|c|}
\hline Heures après vêlage & $\begin{array}{l}\text { Vit. A } \\
\text { U./litre }\end{array}$ & $\begin{array}{l}\text { Vit. A } \\
\text { Y/litre }\end{array}$ & $\begin{array}{c}\text { Carotène } \\
\gamma / 1\end{array}$ & $\begin{array}{c}\text { Matière } \\
\text { grasse } \\
\text { Gr./litre }\end{array}$ & $\begin{array}{c}\text { Vit. A } \\
\text { carotène }\end{array}$ \\
\hline $6 \ldots \ldots \ldots \ldots$ & 2.150 & 750 & 630 & 28 & 1,17 \\
\hline $16 \ldots \ldots \ldots \ldots$ & 2.100 & 740 & 630 & 24,5 & 1,18 \\
\hline $23 \ldots \ldots \ldots \ldots \ldots$ & 1.475 & 512 & 403 & 21 & 1,28 \\
\hline $30 \ldots \ldots \ldots \ldots \ldots$ & 1.410 & 493 & 425 & 24 & 1,16 \\
\hline $40 \ldots \ldots \ldots \ldots \ldots$ & 1.160 & 405 & 325 & 23 & 1,24 \\
\hline $47 \ldots \ldots \ldots \ldots$ & - & 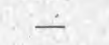 & 188 & 20 & - \\
\hline $54 \ldots \ldots \ldots \ldots \ldots$ & 555 & 194 & 210 & 19 & 0,93 \\
\hline $64 \ldots \ldots \ldots \ldots \ldots$ & 460 & 162 . & 142 & 25 & 1,14 \\
\hline $71 \ldots \ldots \ldots \ldots$ & 144 & 50 & 95 & 30 & 0,5 \\
\hline $78 \ldots \ldots \ldots \ldots$ & - & - & - & 32 & - \\
\hline $88 \ldots \ldots \ldots \ldots$ & 90 & 31,5 & 91 & 18 & 0,35 \\
\hline $95 \ldots \ldots \ldots \ldots \ldots$ & 183 & 64 & 84 & 24 & 0,76 \\
\hline $102 \ldots \ldots \ldots \ldots \ldots$ & 90 & 31,5 & 73 & 21 & 0,45 \\
\hline $184 \ldots \ldots \ldots \ldots \ldots$ & 156 & 54,5 & 77 & 20 & 0,71 \\
\hline $232 \ldots \ldots \ldots \ldots \ldots$ & 100 & 35 & 42 & 20 & 0,83 \\
\hline $246 \ldots \ldots \ldots \ldots \ldots$ & 55 & 19 & 38 & 18 & 0,5 \\
\hline $352 \ldots \ldots \ldots \ldots$ & $1-$ & - & 30 & - & 一 \\
\hline $359 \ldots \ldots \ldots \ldots$ & 39 & 14 & 20 & 18 & 0,7 \\
\hline
\end{tabular}

un taux différent suivant la quantité de carotène contenu dans la ration.

La vitesse de variation est sensiblement la même dans les trois cas. Le colostrum I, bien qu'il soit obtenu au moyen de trois traites par 24 heures, a une composition qui ne varie pas plus vite que celle des autres colostra.

La variation des teneurs en carotène affecte

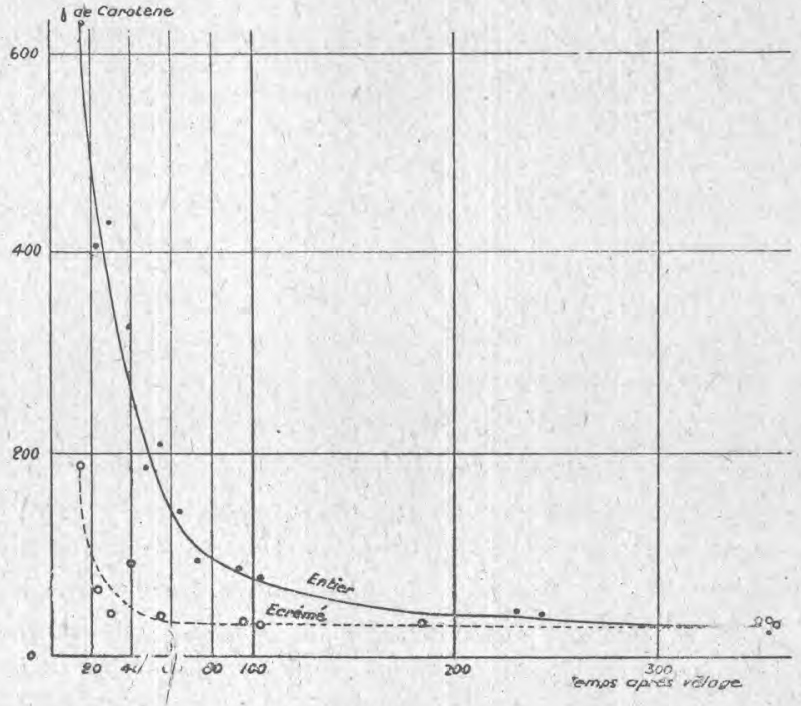

FIG. XIX 
TABLEAU XIII

VACHE NO 3

\begin{tabular}{|c|c|c|c|c|c|c|}
\hline $\begin{array}{c}\text { Heures } \\
\text { après vêlage }\end{array}$ & Jours & $\begin{array}{c}\text { Matière } \\
\text { grasse } \\
\text { Gr./litre }\end{array}$ & $\begin{array}{c}\text { Matière } \\
\text { azotée } \\
\text { Gr./litre }\end{array}$ & $\begin{array}{c}\text { Matière } \\
\text { sèche } \\
\text { Gr./litre }\end{array}$ & $\begin{array}{c}\text { Vitamine } \\
\text { A } \\
\gamma / \text { litre }\end{array}$ & $\begin{array}{c}\text { Carotène } \\
\text { Y/litre }\end{array}$ \\
\hline $5 \ldots$ & - & 44 & 80 & 156 & 1.900 & 2.440 \\
\hline $19 \ldots \ldots \ldots$ & 1 & 28 & 74 & 140 & 1.260 & 2.455 \\
\hline $24 \ldots \ldots \ldots$ & 1 & 57 & 64 & 142 & 740 & 1.490 \\
\hline $96 \ldots \ldots \ldots$ & 4 & 33 & 23 & 118,5 & 550 & 700 \\
\hline $134 \ldots \ldots \ldots$ & 6 & 64,5 & 35 & - & 690 & 615 \\
\hline $182 \ldots \ldots \ldots$ & 8 & 35 & 20,5 & 119,3 & 475 & 335 \\
\hline
\end{tabular}

la même allure que la variation des teneurs en vitamine A.

La courbe XIX montre que la teneur en carotène du colostrum écrémé se fixe rapidement à un taux constant qui rejoint celui du lait écrémé.

\section{E. Carotène et vitamine A par gramme de lipides.}

Les lipides du colostrum écrémé ont la même propriété que ceux du lait écrémé, en ce qui concerne leur pouvoir de fixation du carotène et de la vitamine $\mathrm{A}$. Le colostrum écrémé ( $2^{\mathrm{e}}$ traite) contient près du tiers du carotène total, et $1 / 10^{\mathrm{e}}$ de la vitamine $\mathrm{A}$. 1 gramme de lipides du premier colostrum contient 5 fois plus de carotène qu'un gramme de lipides du produit entier.

Les cénapses lipoprotéiques du colostrum sont donc riches en carotène. Elles perdent cette propriété très rapidement, puisque la courbe XIX montre qu'en 40 heures environ, la teneur en carotène des lipides du colostrum écrémé s'abaissent à celles du lait écrémé. Ces complexes jouent-ils un rôle particulier ?

On sait, depuis les travaux de Famulener [72], Sassenhagen [73] et surtout Sмгтн et Litwle [74] que les anticorps maternels sont transmis au jeune par le colostrum.

Traum [75], Smith et Little ont montré que la transmission de l'immunité ne se fait que dans les deux jours qui suivent la naissance. C'est précisément pendant la même durée que le colostrum écrémé maintient sa teneur élevée en carotène. La chute de la teneur en stérols se produit également dans le même délai.

Il n'est pas sans intérêt de remarquer ce parallélisme, puisque les cénapses lipoprotéiques contiennent du cholestérol en proportion importante, ainsi que du carotène. 
Les cénapses lipoprotéiques du lait semblent par ailleurs contenir des protéines solubles (albumine ou globuline-).

Or, les globulines paraissent être le support des anticorps du colostrum.

(A suivre.)

\title{
PRODUCTION ET CONTROLE HYGIÉNIQUE DU LAIT DANS LES ÉTABLES URBAINES ET SUBURBAINES
}

\author{
par \\ ANDRÉ LOUIS \\ Docteur-Vétérinaire sanitaire
}

(Suite)

\section{$\beta$ RECHERCHE DE L'INTÉGRITÉ de LA MAMELLE}

La recherche de l'intégrité de la mamelle est de la plus haute importance, étant donné la fréquence et la gravité de ses altérations. Seflman, de l'Institut de Laiterie de Kiel, estime que sur deux vaches, il n'y en a qu'une dont le pis soit vraiment sain.

$\mathrm{Au} \mathrm{XI}$ e Congrès international de Médecine Vétérinaire, tenu à Londres en 1930 , F. C. Minetr estime que dans certains grands troupeaux, 60 à $80 \%$ des vaches sont atteints de mammites latentes et qu'en général on peut dire que 15 à $40 \%$ des animaux ont leur mamelle infectée par le streptocoque. "

Les altérations de la mamelle présentent une double importance, hygiénique et économique.

Au point de vue hygiénique, les, laits de mammite peuvent être dangereux pour le consommateur.

Au point de vue économique, le préjudice causé est considérable. D'une part, la quantité de lait produite est très fortement diminuée ; d'autre part, la composition chimique de ces laits est telle que leur mélange avec des laits normaux aboutit à des fabrications industrielles de mauvaise qualité, Nous ne citerons qu'un seul chiffre dont l'éloquence se passe de tout eommentaire. Le 9 janvier 1931, devant la Commission plénière du Landtag prussien, le Docteur Steiger, Ministre de l'Agriculture de Prusse déclarait, que pour cet Etat “les dommages imputables à la mammite streptococcique sont évalués, d'après une estimation prudente, à 250 millions de marks-or par an, soit 1.500 millions de franes français».

\section{SES DIFFICULTÉS}

Lorsqu'on a affaire à une affection tapageuse qui se traduit par des signes cliniques évidents, par une modification notable de la 